\title{
Study on the Instruction Method for Plant Operator
}

\author{
Daiji Kobayashi $^{1}$, Hiroaki Murata ${ }^{2}$, and Sakae Yamamoto ${ }^{2}$ \\ ${ }^{1}$ Nihonbashi Gakkan University \\ 1225-6 Kashiwa Kashiwa-city Chiba, \\ 277-0005 Japan \\ ${ }^{2}$ Tokyo University of Science, \\ 1-3 Kagurazaka Shinjuku-ku Tokyo, \\ 162-8601 Japan
}

\begin{abstract}
In this study, the characteristics of three training curriculums were compared from the viewpoint of the trainee's cognitive process. In the experiment, the nine participants set into three groups of three participants, and trained in each curriculum. In order to evaluate the trainee's cognitive behavior in identifying malfunctions, the concept of mental algorithm was used. As the results, the trainee's cognitive process for identifying malfunctions is estimated as a model. This model could reflect the contents of training curriculum regarding the trainee's cognitive process.
\end{abstract}

Keywords: plant, operator, instruction, thinking process.

\section{Background}

For a plant operator, it is important to find out the cause of plant disturbance. When the plant's components have some trouble, the plant operators have to identify the troubled situation according to some procedural manuals, documented instructions and so on. However, our experimental studies indicated that the operator's empirical techniques affect their subtle thinking for identifying malfunctions. Kobayashi (2003) investigated the thinking process including subtle thinking through some simulator experiment, and named the characteristic thinking process mental algorithm. The mental algorithm is represented by the sequence of subtle thinking steps. Each step of subtle thinking, occurs between getting information from the interface, is mainly composed of cognitive state and strategy. Therefore, the transition of the cognitive state and the contents of strategy could represent the operator's cognitive behavior. From this point, Kobayashi evaluated some types of plant interfaces (the screens on CRT display) experimentally.

The plant operator is used to learn the techniques for the practical operation task in accordance with their experiences. The techniques, of course, are not only for appropriate action but also for cognitive behavior. The cognitive behavior which would be observed by the mental algorithm is the product of experiences, instructions, trainings, and so on. In case of Japanese nuclear power plant, the training curriculums for the operators are systematized through trial and error to some extent. The reason 
why it is difficult to pursue more appropriate training curriculum is based on two facts owing to the variation of the plant and the human operator as follows:

First, some types of nuclear power plant has been developed with the advancement of technology in Japan, then some types of full-scale simulator has been used for operator training. In case of BWR power plant, there are three types of BWR power plants, and three types of full-scale simulator are used. Moreover, the specifications of the same type of the BWR power plants are slightly different each other, therefore the trainer have to take into account the variation especially for the simulator training.

Next, it is worried about to train up the successors for old plants, because Japanese face the serious concerns of rapid aging and very low birthrate. Many veteran operators, are baby boomers, reach the retirement age, have great deal of experiences and skills. These experiences and skills could not represent by words, therefore it is difficult to hand their experiences and skills on the next generation as early as possible.

Concerning about the above facts, the operator training curriculums for BWR power plant should be re-considered based on their appropriateness.

Previous studies proposed some model representing the relation between the operator's skill and their cognitive behavior. For example, Rasmussen's SRK-model is a pioneering work, and GEMS dynamic model proposed by Embrey and Reason (1986) is concretized and detailed SRK-model. On the other hand, Yukimachi and Hasegawa (1999) revised SRK-model in order to analyze the human errors at nuclear power plant in Japan. In these models, the relation between the operator's skill level and cognitive behavior is represented based on some observational studies and example analysis of human errors. However, it is difficult to use that knowledge to make the training curriculum, and we tried another approach to the evaluation of the training curriculum from cognitive viewpoint.

Although our goal is to develop the method of evaluation for instruction and training curriculum, we tried to investigate the relation between training curriculum and the characteristics of the operator's thinking process for identifying malfunctions as the first step of our research. Therefore, the object of this paper is to reveal the cognitive mechanism of the instruction, and the training effect on operator's thinking process for identifying malfunctions.

\section{Method}

In order to compare the cognitive processes of each participant, we conducted the same simulator experiment after training by different three curriculums of each groups. In the experiment, the number of cognitive failure and the types of the cognitive failure were observed based on their performances and verbal protocols.

\subsection{Apparatus}

The participants' verbal protocols and their performances were recorded by a video camera, and the participant's eye tracking was recorded by an eye mark analyzer (NAC Image Tech. EMR-8) using a simulator after the training. The simulator was composed of PC and 22-inches touch monitor (see Figure 1). Figure 2 shows the 
screen of the touch monitor indicating the part of a nuclear power plant's process, 12 annunciators, and 2 indicators for selected component. The indicators on the lower part of the screen are a line chart and a parameter list. These are updated every second. In addition, each component's main parameter on the upper part is also updated every second. When a participant wants to refer a component's all parameters, the participant has to select and touch the component icon on the screen using a stick. The simulator records these historical operation data in its hard disk.

\subsection{Participants}

Participants were nine male Japanese university students. They did not have any knowledge about the plant operation; however they have the knowledge about basic thermodynamics. In order to compare the participant's cognitive behavior in simulator experiment, we set the nine participants into three group (Group-A, -B, -C) of three participants, and trained in each curriculum.

\subsection{Training}

Firstly, an experimenter lectured all participants on the way of simulator operation and basic knowledge about the process for supervising. Mainly we told a behavior and dynamics of components.

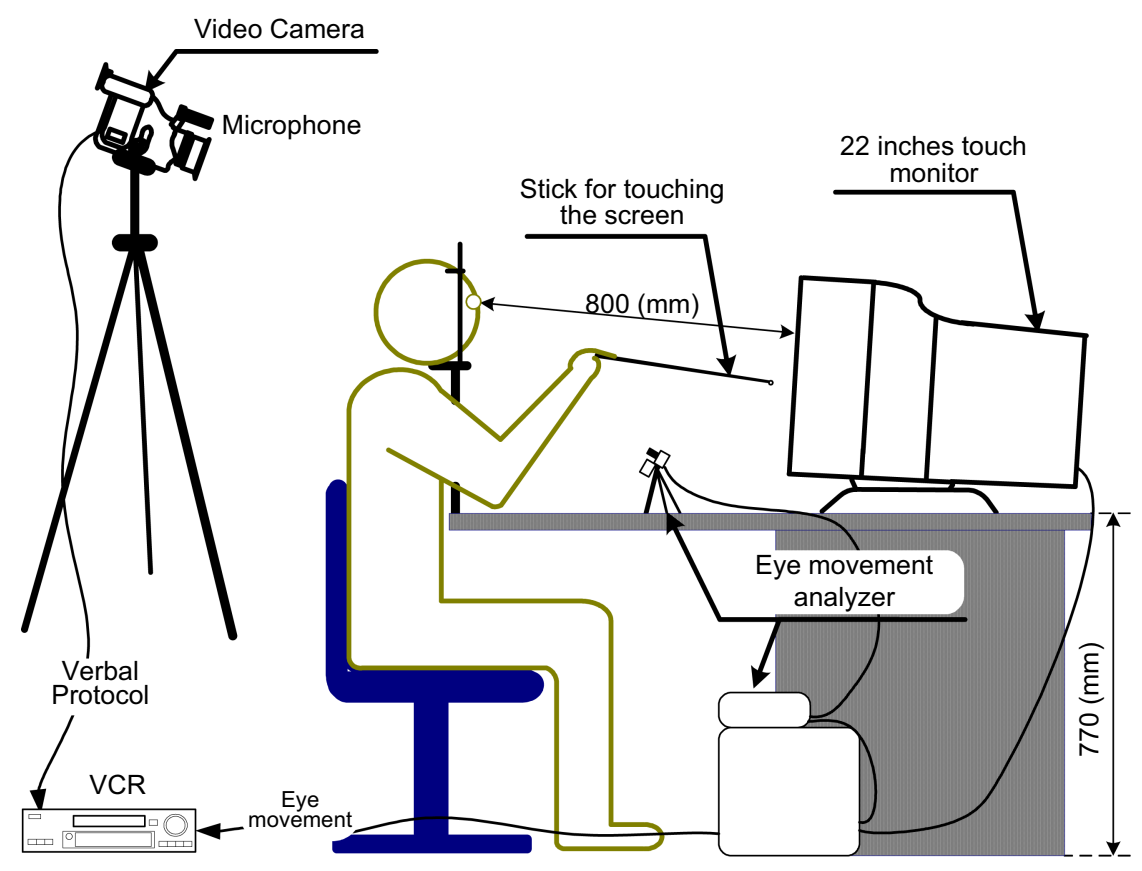

Fig. 1. Experimental apparatus 


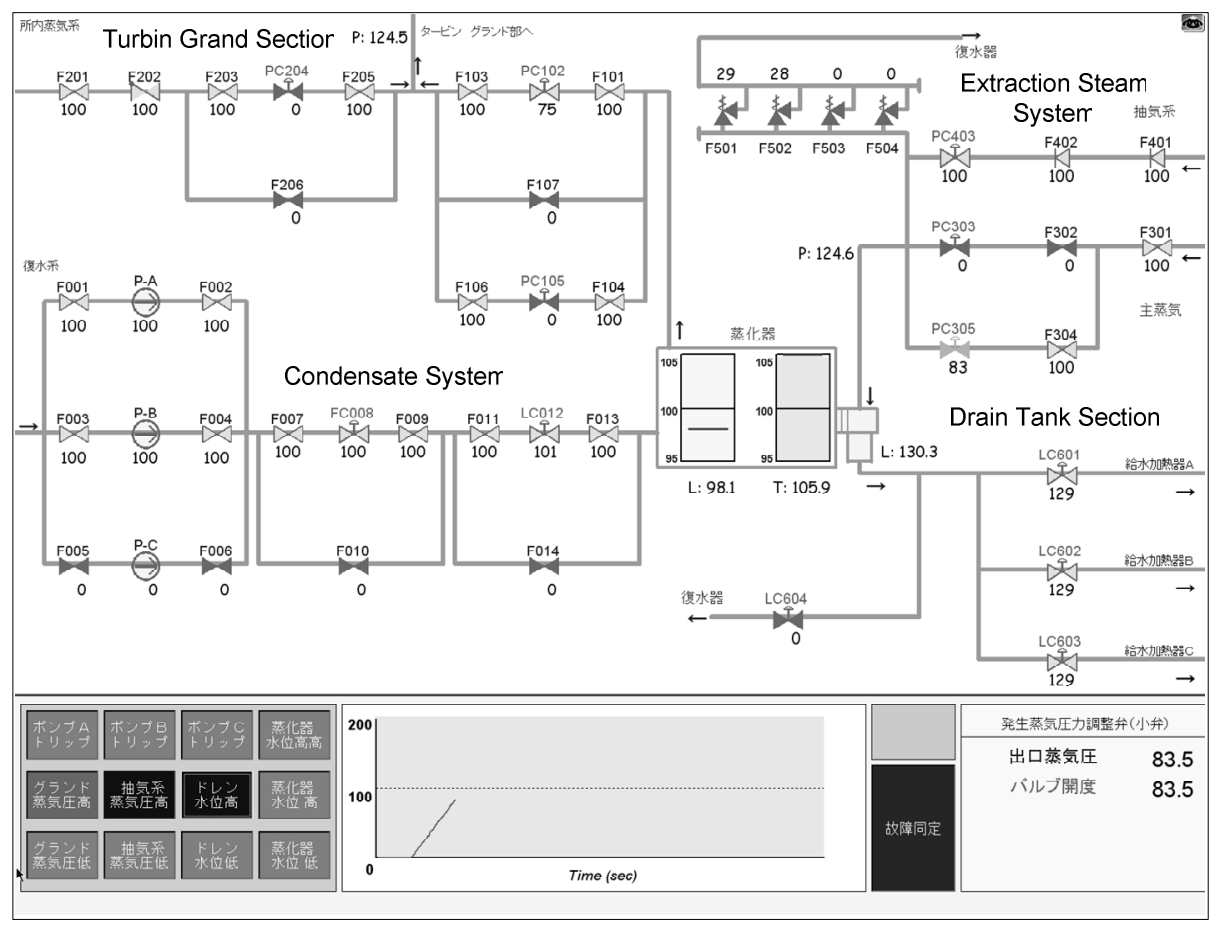

Fig. 2. Contents of the simulator's screen on CRT

Next, the experimenter instructed how a malfunction affects the other components' parameter.

Last, the experimenter trained the participants in the following different way: In case of Group-A, the participants underwent simulator training using ten scenarios including a malfunction of each; To the participants belonging to Group-B, the experimenter lectured on the empirical way of identifying malfunctions in order to make them obtain some rule-based way of thinking. After the lecture, they underwent same simulator training; In case of Group-C, we instructed the participants to think or image the transitional parameter of some components after a malfunction occurred in order to make them have the image of each component's transitional state. After the instruction, they underwent the simulator training in the same way.

\subsection{Simulator Experiment}

After the simulator trainings, we conducted simulator experiments using fifteen scenarios including a few malfunctions. Each participant tried to identify all malfunctions in each scenario, and tried to speak their thinking process while a trial. Their performance and verbal protocols were recorded by a video camera, and the component's name and the time they referred was recorded by PC. After the experiment, we heard about the participant's opinion about the trials. 
Table 1. Comparison of curriculum contents for three groups

\begin{tabular}{|c|c|c|c|}
\hline \multirow{2}{*}{ Curriculum contents } & \multicolumn{3}{|c|}{ Group } \\
\hline & A & B & C \\
\hline $\begin{array}{l}\text { Instruction by lecturing using slides on the followings: } \\
\text { - Outline of the process } \\
\text { - Each component behavior in the processes }\end{array}$ & 1st Day & 1st Day & 1st Day \\
\hline Paper test for the understanding of the above lecture & 2nd Day & 2nd Day & 2nd Day \\
\hline $\begin{array}{l}\text { Instruction on the diagnosis methods from the component } \\
\text { behavior using the simulator and a manual }\end{array}$ & $\mathrm{N} / \mathrm{A}$ & \multirow{2}{*}{ 3rd Day } & $\mathrm{N} / \mathrm{A}$ \\
\hline $\begin{array}{l}\text { Demonstration of the identifying malfunctions } \\
\text { using the simulator }\end{array}$ & 3rd Day & & \multirow{2}{*}{ 3rd Day } \\
\hline $\begin{array}{l}\text { Training of the reasoning skill in estimation of malfunctions } \\
\text { using the simulator's still screen }\end{array}$ & $\mathrm{N} / \mathrm{A}$ & $\mathrm{N} / \mathrm{A}$ & \\
\hline $\begin{array}{l}\text { Training of identifying malfunctions included } 10 \text { scenarios } \\
\text { using the simulator }\end{array}$ & 4th Day & 4th Day & 4th Day \\
\hline
\end{tabular}

N/A: not applicable

\section{Result}

As the consequence of analyzing the participants' verbal protocols, some cognitive failures in their thinking process were found. The cognitive failures were, for example, misunderstanding, decision error, overlooking, and so on.

\subsection{Cognitive Failure}

The averaged number of cognitive failure was 8.3 per participant of the Group A. The most of these cognitive failures were to be confused in determination of the component's state. Therefore, the failures could be caused by the lack of thinking skill for identifying malfunction.

The Group B's averaged number of cognitive failures was 4.7 per participant. Most of the cognitive failures were occurred by "one-track way thinking" which would be caused by using inappropriate rules for thinking.

The Group C's averaged number of cognitive errors was 3.0.per participant. Most of the cognitive failures were occurred by careless oversight or perceived notion. In addition, it was found that a participant was not able to acquire the way to identify malfunction sufficiently.

The contents of participants' cognitive failure could be categorized as shown in Table 2.

Table 2 indicates that the cognitive failure by the participants of Group A occurred more frequently than the other groups' cognitive failures, especially the participants of Group-A tended to fail on the stage of grasping the situation in the identification process. 
Table 2. Cognitive failures of each participant in each group

\begin{tabular}{|c|c|c|c|c|c|c|c|c|c|c|}
\hline \multirow{2}{*}{\multicolumn{2}{|c|}{$\begin{array}{rr} & \text { Group } \\
\text { Cognitive failure } & \text { Perticipant }\end{array}$}} & \multicolumn{3}{|c|}{ A } & \multicolumn{3}{|c|}{ B } & \multicolumn{3}{|c|}{$\mathrm{C}$} \\
\hline & & $\mathrm{a}$ & $\mathrm{b}$ & c & d & $\mathrm{e}$ & $f$ & $\mathrm{~g}$ & $\mathrm{~h}$ & 1 \\
\hline \multirow{4}{*}{ 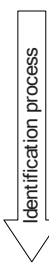 } & $\begin{array}{l}\text { Oversights of parameter changes indicating the } \\
\text { unusual process condition }\end{array}$ & 4 & 3 & 1 & 2 & 2 & 0 & 1 & 1 & 0 \\
\hline & Mistaking component's conditions & 2 & 2 & 3 & 2 & 0 & 2 & 1 & 1 & 0 \\
\hline & Mistaken decision about malfunction & 2 & 1 & 1 & 1 & 2 & 2 & 2 & 1 & 2 \\
\hline & $\begin{array}{l}\text { Confusion on deducting the state of the process } \\
\text { from the components' condition }\end{array}$ & 2 & 4 & 0 & 0 & 0 & 1 & 0 & 0 & 0 \\
\hline & Total & 10 & 10 & 5 & 5 & 4 & 5 & 3 & 3 & 2 \\
\hline
\end{tabular}

\subsection{Mental Algorithm}

In order to investigate the reason why the frequency of cognitive failure varies, the mental algorithm of participants in the simulator experiment was estimated based on their verbal protocols and performances. Previous studies evaluated the operator's thinking process based on the transition of cognitive states from their perception about the plant disturbance to their identification about the malfunctions of those. Therefore, the reason of the cognitive failures was investigated through the transition of cognitive state.

From the result of the simulator experiment, we found that the transition of the cognitive state occurred whenever the operator gets the component's state by touching the component's icon on screen. From this point, we estimated the cognitive state, counted the transition pattern of the cognitive state on a group, and calculated the transition pattern's ratio of each group. As the result, four types, that are confusing, lack direction, having some directions, and having an orientation with a clear direction, categorized as the cognitive states.

Table 3 shows the transition pattern's ratio of the cognitive state and the number of transition to each cognitive state. The number of transition is the number of getting a component state. Therefore, Table 3 indicates the Group-A's participants search more

Table 3. Transition pattern of cognitive state in each group (left side: Group-A, middle: Group$\mathrm{B}$, right side: Group-C)

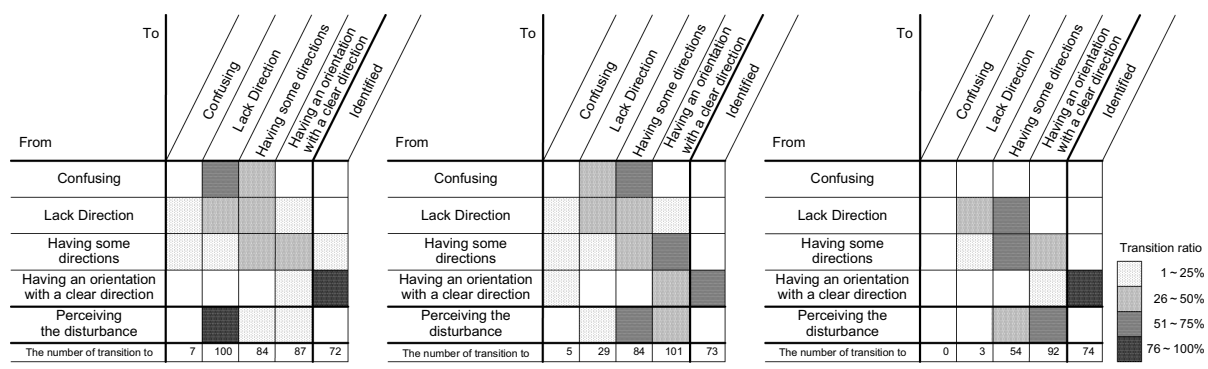




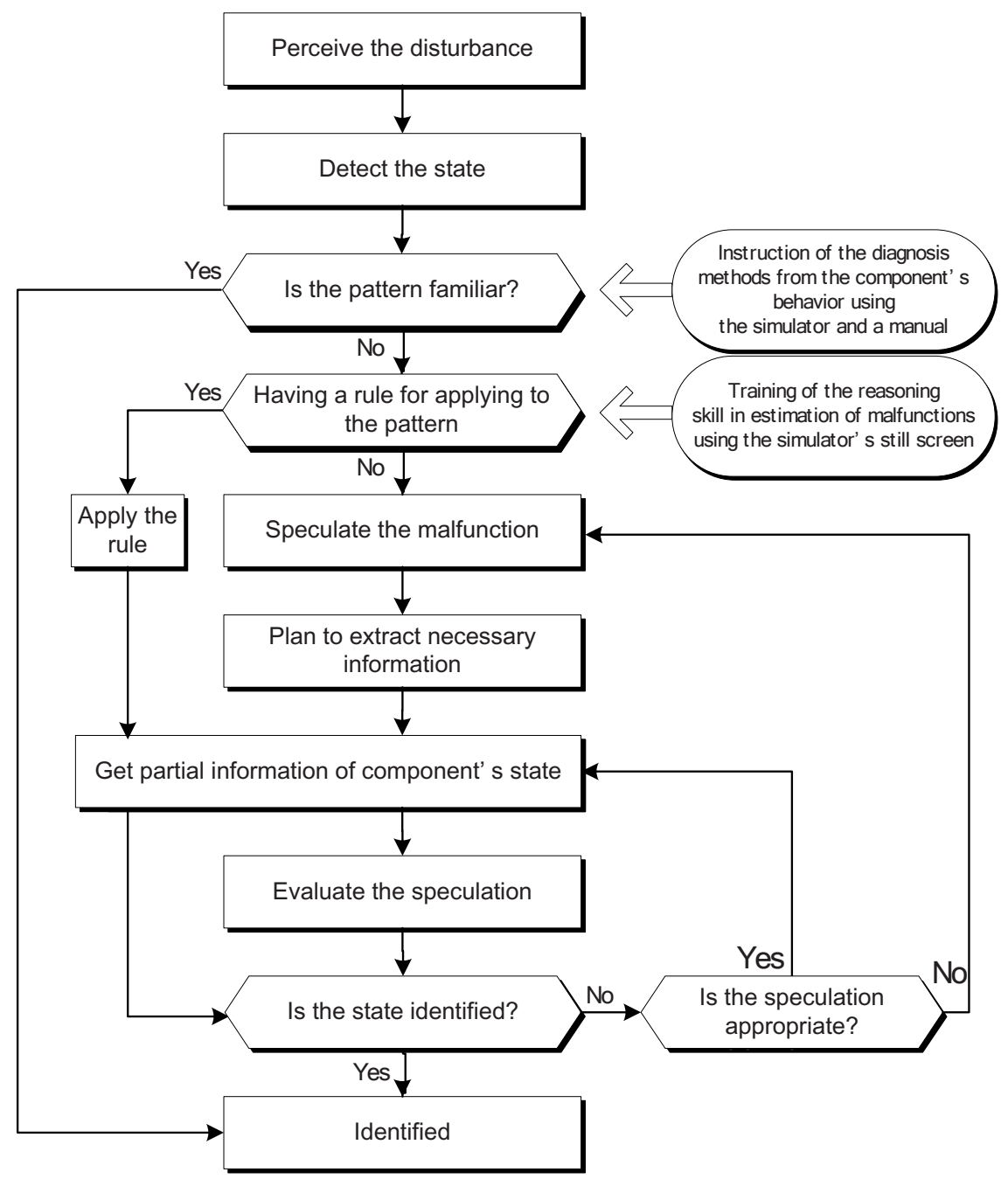

Fig. 3. Trainee's cognitive model for identifying malfunctions

components than the others. Further, the number of transition to confusing or lack direction is also more than the others are. This means the Group-A's participants could not obtain information for identifying malfunctions in spite of the trainings.

In case of Group-B, the number of transition is higher than Group-C's, and transition to confusing and lack direction is more frequently than Group-C's. Therefore, their identifying process could not be sufficient.

From these results, we found that the Group-C's cognitive condition is the best cognitive condition in three groups we tried. 


\section{Discussion}

The results of the mental algorithm analysis indicates that Group-A's participants could not obtain the way of identifying malfunctions sufficiently. Therefore, the number of cognitive failures would be more than the other groups'. In other words, it is necessary for the Group-A to learn the diagnosis methods from the component's behavior on screen.

The group-C's participants could not speculate logically but could depend on our rules that we had instructed in advance. Therefore, these results represent that it is important for the operators to understand the mechanism of plant's process.

From above of these investigations, the trainee's cognitive process for identifying malfunctions is estimated as Figure 3. This model reflects the contents of training curriculum regarding the trainee's cognitive process.

\section{Conclusion}

We compared three types of the training methods and investigated it from cognitive process. Consequently, we could indicate the relation between the characteristics of these trainings and the cognitive process.

\section{References}

1. Kobayashi, D., Yamamoto, S.: Experimental study of evaluating graphical CRT displays in process plants based on operator's thinking process. The Journal of Japan Ergonomics Society, Vol. 37, No. 3, 113-124 (2001)

2. Sakata, K., Nishitani, H.: Considerations on Simulator Training for Plant Operations during Malfunctions. The Journal of the Society for Industrial Plant Human Factors of Japan, Vol. 5, No. 2, 130-140 (2000)

3. Sheridan, T.B.: HCI in Supervisory Control: Twelve Dilemmas. Lecture Notes in Control and Information Sciences, Vol. 253, 1-12 (2000)

4. Stammers, R.B.: Training issues: Computer Science Today. Human Factors in Nuclear Safety, 189-196 (1996)

5. Yukimachi, T., Hasegawa, T.: Analysis of human errors at nuclear power plants in Japan. The Journal of the Society for Industrial Plant Human Factors of Japan, Vol. 4, No. 1, 48-57 (1999) 